Rafael ISOLAN FEREZIN ${ }^{1}$

Dennis ARMANDo Bertolin ${ }^{2}$

Izabel Galhardo Demarchi

\title{
Prevalência de sorologia positiva para HIV, hepatite B, toxoplasmose e rubéola em gestantes do noroeste paranaense
}

\author{
Prevalence of positive sorology for HIV, hepatitis B, toxoplasmosis \\ and rubella in pregnant women from the northwestern region of the \\ state of Paraná
}

Artigo Original

Palavras-chave

Soropositividade para HIV Transmissão vertical de doença infecciosa Hepatite B/congênito

Toxoplasmose/congênito Rubéola (sarampo alemão)/congênito

Diagnóstico pré-natal

Gestantes

Keywords

HIV soropositivity

Infectious diseases transmission, vertical Hepatitis B/congenital Toxoplasmosis, congenital Rubella/congenital Prenatal diagnosis Pregnant woman

\section{Resumo}

OBJETIVO: Verificar a soroprevalência do vírus da imunodeficiência humana (HIV), hepatite B (VHB), toxoplasmose e rubéola em gestantes do noroeste paranaense. MÉTODOS: Foi realizado um estudo retrospectivo a partir dos resultados de exames sorológicos de triagem no pré-natal de 1.534 pacientes atendidas durante o primeiro semestre de 2010. Foram incluídos somente resultados do primeiro exame de pré-natal e aqueles com pesquisa simultânea de $\lg G$ e $\lg M$ para toxoplasmose e rubéola. A sorologia foi realizada por enzimaimunoensaio em micropartículas (MEIA). Para análise estatística foi empregado o teste do $\chi^{2}$, com nível de significância de 5\%. RESULTADOS: A positividade para $\circ$ HIV foi de $0,3 \%$, a sorologia para $\mathrm{VHB}$ pelo marcador $\mathrm{HBsAg}$ foi positiva em $0,5 \%$ das gestantes, enquanto a reatividade para anticorpos IgM anti-Toxoplasma gondii foi de 1,1\% e para lgG de 59\%. Em relação à rubéola nenhuma sorologia mostrou positividade para lgM, e para lgG a reatividade foi de $99,6 \%$. A análise dos resultados mostrou que não há associação entre as soroprevalências estudadas e a idade das pacientes, exceto quanto à frequência de lgG anti-T. gondii, que foi mais elevada na faixa etária entre 30 e 44 anos. CONCLUSÃO: A soroprevalência dessas doenças infecciosas em gestantes do noroeste do Paraná é compatível com outras regiões do Brasil.

\section{Abstract}

PURPOSE: To ascertain the seroprevalence of human immunodeficiency virus (HIV), hepatitis B (HBV), toxoplasmosis and rubella infections in pregnant women in northwestern Paraná. METHODS: We conducted a retrospective study based on the results of serological screening during prenatal care of 1,534 patients during the first half of 2010. We included only results from the first prenatal exam and with a simultaneous search for $\lg G$ and $\lg M$ antibodies to rubella and toxoplasmosis. Serology was performed by microparticle enzyme immunoassay (MEIA). Data were analyzed statistically by the $\chi^{2}$ test, with the level of significance set at $5 \%$. RESULTS: HIV positivity was $0.3 \%$, positivity of HBV serology $(\mathrm{HbsAg})$ was $0.5 \%$, reactivity to IgM antibodies to Toxoplasma gondii was $1.1 \%$, and reactivity to IgG antibodies was $59.0 \%$. For rubella, no patient was positive for $\lg M$, and $\lg G$ reactivity was $99.6 \%$. Data analysis showed no statistical association between seroprevalence and patient age, except for the frequency of anti-T. gondii lgG, which was higher in the 30 to 44 year age group. CONCLUSION: The prevalence of these infectious diseases in pregnant women from northwestern Paraná is comparable to that observed in other regions of Brazil.
Correspondêncio Izabel Galhardo Demarch Universidade Estadual de Maringa Avenida Colombo, 5790 bloco -90 , sala 3 - Zona 7 CEP: 87020-900

Maringá (PR), Brasi Recebido em 19/07/2012

Aceito com modificacões 12/12/2012
Trabalho realizado no Departamento de Análises Clínicas e Biomedicina, Universidade Estadual de Maringá - UEM - Maringá (PR), Brasil. 'Programa de Pós-graduação de Biociências Aplicadas à Farmácia, Universidade Estadual de Maringá - UEM - Maringá (PR), Brasil. 2Departamento de Análises Clínicas e Biomedicina, Universidade Estadual de Maringá - UEM - Maringá (PR), Brasil. Conflito de interesses: não há 


\section{Introdução}

No Brasil, a qualidade e a humanização do atendimento à gestante são enfoques essenciais na atenção obstétrica e neonatal realizada pelo Sistema Único de Saúde (SUS) ${ }^{1}$. Visando assegurar o nascimento de uma criança saudável e garantir o bem-estar de mãe e filho, estão inclusos na atenção pré-natal exames laboratoriais que detectam os principais agravos e riscos gestacionais ${ }^{1}$. Os exames sorológicos rotineiramente realizados para diagnóstico de doenças infecciosas de acordo com preconização pelo Ministério da Saúde (MS) incluem o Veneral Diseases Research Laboratory (VDRL) para diagnóstico da sífilis, testagem para o Vírus da Imunodeficiência Humana (HIV), pesquisa de IgG e IgM para toxoplasmose e pesquisa do antígeno de superfície do vírus da hepatite $\mathrm{B}$ (HBsAg). A pesquisa para rubéola é indicada quando há sintomatologia sugestiva ${ }^{1}$.

A triagem sorológica para essas doenças é de suma importância na gestação, pois possibilita o tratamento precoce, evitando a ocorrência de malformações congênitas e/ou problemas tardios relacionados à hepatite $\mathrm{B}$, toxoplasmose e síndrome da rubéola congênita (SRC) ${ }^{2-5}$. Quando detectado o HIV em gestante, o tratamento durante a gestação inibe em até $99 \%$ a transmissão materno-fetal do vírus. No caso de hepatite $B$, se a gestante for tratada com imunoglobulina contra o vírus da hepatite $\mathrm{B}$ e a criança receber a vacina nas primeiras doze horas após o nascimento, a transmissão congênita pode ser evitada de 85 a $95 \%{ }^{4,6}$. As medidas profiláticas para evitar-se a toxoplasmose congênita incluem a realização da triagem sorológica no pré-natal e, uma vez diagnosticada a doença em fase aguda, submeter a gestante à terapia medicamentosa imediata, a qual pode reduzir significativamente a transmissão vertical ${ }^{7-10}$. A rubéola congênita pode ser evitada através de vacinação prévia e por medidas de higiene preventiva ${ }^{11}$.

A soroprevalência dessas infecções em gestantes varia nas diferentes regiões do Brasil ${ }^{12-16}$. Há poucos estudos realizados no Estado do Paraná12,17; no entanto não foram encontradas publicações sobre triagem sorológica em gestantes do noroeste desse Estado. Portanto, o objetivo deste estudo foi verificar a soroprevalência ao HIV, hepatite $\mathrm{B}$, toxoplasmose e rubéola em gestantes nessa região do Estado.

\section{Métodos}

Realizou-se um estudo retrospectivo, no qual foram levantados a idade e os resultados de testes sorológicos utilizados no diagnóstico para HIV, VHB, toxoplasmose e rubéola de 1.534 gestantes provenientes de 29 municípios pertencentes à $15^{\text {a }}$ Regional de Saúde do Paraná. A idade das gestantes variou entre 14 e 44 anos, com média de 25,1 anos e desvio padrão de 6,5 anos. Das 1.534 pacientes envolvidas neste estudo, $354(23,0 \%)$ enquadraram-se na idade de 14 a 19 anos, $813(53,0 \%)$ de 20 a 29 anos e $367(24,0 \%)$, de 30 a 44 anos.

As pacientes foram atendidas no Laboratório de Ensino e Pesquisa em Análises Clínicas (LEPAC), da Universidade Estadual de Maringá (UEM), entre $1^{\circ}$ de janeiro e 31 de junho do ano de 2010. Foram incluídos apenas os resultados de exames realizados no primeiro atendimento pré-natal.

As pacientes encaminhadas ao LEPAC são oriundas do SUS, as quais tiveram solicitação médica para exames de pré-natal. No laboratório foi feita a coleta de amostras de sangue para obtenção de soro e realização dos exames solicitados, cujos resultados foram repassados às pacientes para tratamento e/ou orientação médica.

A dispensa do Termo de Consentimento Livre e Esclarecido foi permitida pelo Comitê Permanente de Ética em Pesquisa Envolvendo Seres Humanos (COPEP) da Universidade Estadual de Maringá, n ${ }^{\circ}$ do parecer 550/11.

O diagnóstico das infecções por HIV (triagem), VHB, Toxoplasma gondii e vírus da rubéola foi realizado por sorologia utilizando a tecnologia de enzimaimunoensaio em micropartícula (MEIA), automatizada em equipamento AxSYM (Abbott Laboratories, Abbott Park, Illinois), conforme as recomendações do fabricante.

As amostras foram consideradas reativas para anticorpos anti-HIV quando a razão amostra sobre cut-off (S/CO) foi $\geq 1,00$; hepatite $\mathrm{B}$ quando $\mathrm{S} / \mathrm{CO} \geq 2,00$; para toxoplasmose IgG, amostras foram consideradas positivas quando $\mathrm{S} / \mathrm{CO} \geq 3,0 \mathrm{UI} / \mathrm{mL}$ e inconclusivas quando entre $2,0 \mathrm{e}$ $2,9 \mathrm{UI} / \mathrm{mL}$, para IgM positivas quando $\mathrm{S} / \mathrm{CO} \geq 0,600 \mathrm{UI} / \mathrm{mL}$ e inconclusivas quando entre 0,500 e $0,599 \mathrm{UI} / \mathrm{mL}$. As amostras identificadas com resultado positivo ou inconclusivo para toxoplasmose foram encaminhadas para o Laboratório Central do Paraná (LACEN-PR) para a realização do teste de avidez de IgG, cujo resultado não tivemos acesso. Para rubéola, os resultados de $\operatorname{IgG}$ foram considerados positivos quando $\geq 10,0 \mathrm{UI} / \mathrm{mL}$ e inconclusivos quando entre 5,0 e 9,9 UI/mL. Já IgM, foram positivas as amostras com $\mathrm{S} / \mathrm{CO} \geq 0,800 \mathrm{UI} / \mathrm{mL}$ e inconclusivas quando entre 0,600 e $0,799 \mathrm{UI} / \mathrm{mL}$. Os pacientes com resultado inconclusivo para rubéola não tiveram interpretação laboratorial.

Nos testes de toxoplasmose e rubéola, os resultados positivos para IgG acompanhados de resultado negativo para $\operatorname{IgM}$ foram considerados indicativos de cicatriz sorológica e imunização à doença: os do tipo $\operatorname{Ig} \mathrm{M}$ foram considerados indicativos de infecção recente independente de o resultado de IgG ser positivo ou negativo. Somente os resultados de sorologia com pesquisa simultânea de $\operatorname{IgG}$ ou $\operatorname{Ig}$ M foram incluídos nesse estudo. 
Para a comparação da distribuição da variável faixa etária e a soroprevalência, foi utilizado o teste do $\chi^{2}$ ou teste exato de Fisher usando o software STATA 9.1, com intervalo de confiança de 95\%. A Odds Ratio (OR) foi utilizada para avaliar a força de associação entre a variável faixa etária e a soroprevalência.

\section{Resultados}

Do total de gestantes incluídas, 1.508 foram submetidas ao teste anti-HIV e quatro $(0,3 \%)$ tiveram o resultado reagente, e não se mostrou associação estatística com a idade. A pesquisa de HBsAg foi realizada em 1.483 gestantes e a frequência de positivos foi de $0,5 \%$ (oito gestantes), porém em relação à faixa etária não houve diferença significativa na distribuição das pacientes (Tabela 1).

Com relação à toxoplasmose, em 97,7\% (1.498) das gestantes foi examinado o anticorpo $\operatorname{IgG}$ e o $\operatorname{IgM}$, com positividade de $59 \%$ (883) e 1,1\% (16 casos), respectivamente. Houve diferença significativa entre as faixas etárias quanto à reatividade para IgG anti-Toxoplasma gondii, que se mostrou mais frequente em pacientes com idade entre 30 e 44 anos $(\mathrm{p}<0,001)$ e de 20 a 29 anos $(\mathrm{p}<0,001)$. $\mathrm{Na}$ faixa etária dos 30 aos 44 anos, a frequência de IgG anti-T. gondii foi de $69,6 \%$, enquanto entre as pacientes de 20 e 29 anos foi de 59,4\% e, entre 14 a 19 anos, foi de $46,8 \%$. As gestantes com idade de 30 a 44 anos tiveram uma probabilidade três vezes maior de ter IgG positiva para toxoplasmose em relação às mais jovens. Para IgM anti-T. gondii não houve diferença significativa quanto a esse aspecto (Tabela 1), permanecendo as três faixas etárias com negatividade superior a 97\%. Das 16 pacientes $\operatorname{Ig} \mathrm{M}$ positivas, 14 também tinham positividade para IgG, uma apresentou resultado inconclusivo (zona cinza) e outra resultado negativo para essa classe de anticorpo.

Quanto à sorologia para rubéola, 223 gestantes foram testadas e a frequência de IgG foi de 99,6\% (222) e não houve casos positivos para IgM. Nenhuma faixa etária demonstrou diferença significativa quanto à reatividade dos testes, tanto para IgG quanto para IgM (Tabela 1).

\section{Discussão}

Este estudo detectou uma frequência de $0,3 \%$ de sorologia positiva para o HIV nas gestantes testadas do noroeste do Paraná. Em Londrina, ainda no Paraná, verificou-se $0,6 \%{ }^{12}$ de positividade para o HIV; Campo Grande (MS), 0,2\% ${ }^{13}$;São José do Rio Preto (SP), 2,1\% ${ }^{14}$; Sergipe $0,1 \%^{15}$; Caxias do Sul (RS) 2,7\% ${ }^{16}$; e na Amazônia ocidental, $0,6 \%{ }^{17}$. Esses estudos referidos realizaram a pesquisa de HIV pela técnica de enzimaimunoensaio (ELISA) e nenhum evidenciou relação entre a frequência do anticorpo e a faixa etária.

Devido à eficácia das medidas disponíveis para evitar a infecção pelo HIV, atualmente a Organização Mundial da Saúde considera uma meta realista a total eliminação da ocorrência de novas infecções e mortes relacionadas ao vírus. Portanto, o diagnóstico no pré-natal é imprescindível no intuito de monitorar a existência de gestantes soropositivas e evitar infecções congênitas ${ }^{2}$.

$\mathrm{O}$ índice de positividade de $0,5 \%$ para hepatite $\mathrm{B}$ se assemelha aos relatos da literatura que mostram $0,8 \%$ de positividade para VHB em Londrina ${ }^{12}, 0,3 \%$ em Campo Grande $^{13}, 0,5 \%$ em Goiânia $(\mathrm{GO})^{18}, 0,6 \%$ em Recife $(\mathrm{PE})^{19}, 1,8 \%$ em São José do Rio Preto ${ }^{14}$ e $0,7 \%$ na Amazônia ocidental ${ }^{17}$, todos esses estudos utilizaram a técnica de ELISA. Como a infecção neonatal por hepatite B gera mais risco de cronificação da doença e evolução para cirrose e hepatocarcinoma ${ }^{3}$, e diante da persistente prevalência da infecção entre a população, é extremamente importante realizar a sorologia de triagem no pré-natal a fim de iniciar o tratamento precocemente, ou mesmo evitar a transmissão vertical ${ }^{4}$.

Os resultados sorológicos para toxoplasmose demonstraram positividade baixa para anticorpos do tipo $\operatorname{IgM}$ e alta para IgG. Em Londrina observou-se 1,8\% de positividade para $\operatorname{IgM}$ e $67 \%$ para $\mathrm{IgG}^{12}$; em Cascavel no Paraná, a prevalência de IgG foi de $54 \%$ e $\operatorname{IgM}$ de $1,2 \%{ }^{20}$; em Campo Grande de 0,4\% para IgM e 91\% para $\operatorname{IgG}^{13}$; em Goiânia de $0,7 \%$ para IgM e de $67,7 \%$ para $\operatorname{IgG}^{21}$; no Estado de Sergipe 0,4\% para IgM e 69,3\% para IgG ${ }^{15}$; em São José do Rio Preto 3,4\% para $\operatorname{IgM}$ e $62 \%$ para $\operatorname{IgG}^{14}$ e; em Caxias do Sul verificou-se frequência de $\operatorname{IgM}$ de

Tabela 1. Soroprevalência ao HIV, hepatite B, toxoplasmose e rubéola em gestantes do noroeste do Paraná segundo a faixa etária, testadas no primeiro semestre de 2010

\begin{tabular}{|c|c|c|c|c|}
\hline & $14-19$ & $20-29$ & $30-44$ & Total \% \\
\hline HIV & 0,3\% (OR: 0,$4 ;$ IC95\% 0,0-6,9) & $0,1 \%$ (OR: 1,0; IC95\% 1,0) & $0,1 \%$ (OR: 0,$2 ;$ IC95\% 0,0-2,5) & 0,3 \\
\hline Hepatite B & 0,6\% (OR: 0,9; IC95\% 0,2-4,8) & 0,5\% (OR: 1,0; IC95\% 1,0) & 0,6\% (OR: 0,$9 ;$ IC95\% 0,2-4,9) & 0,5 \\
\hline Toxo (lgG) & 46,8\% (OR: 1,0; IC95\% 1,0) & 59,4\% (OR: 1,7; IC95\% 1,3-2,2*) & $69,6 \%$ (OR: 2,6; IC95\% 1,9-3,6*) & 59 \\
\hline Toxo (IgM) & 0,9\% (OR: 0,9; IC95\% 0,2-3,5) & 0,8\% (OR: 1,0; IC95\% 1,0) & 2,0\% (OR: 0,4; IC95\% 0,1-1,2) & 1,1 \\
\hline Rubéola (IgG) & $98,3 \%$ & $100 \%$ & $100 \%$ & 99,6 \\
\hline Rubéola (IgM) & $0 \%$ & $0 \%$ & $0 \%$ & 0 \\
\hline
\end{tabular}

OR: Odds Ratio; IC95\%: intervalo de confiança de 95\%.

${ }^{*} p<0,001$ (teste do $\chi^{2}$ ). 
$1,8 \%^{16}$. Isso demonstra que entre as gestantes incluídas neste estudo há uma menor frequência de contato prévio com o T. gondii em relação a algumas regiões do país, como Sergipe e Campo Grande. Além disso, vale ressaltar que esses estudos utilizaram a técnica de ELISA.

Em relação à faixa etária, semelhante ao estudo atual, em Sergipe foi relatado um aumento da prevalência do anticorpo IgG anti-T.gondii com o aumento da idade ${ }^{15}$. A toxoplasmose congênita pode ser evitada com terapia medicamentosa específica durante a gestação, o que previne as graves consequências para a criança ${ }^{5}$.

No presente estudo, a sorologia para rubéola não detectou nenhuma paciente positiva para $\operatorname{IgM}$ e a maioria possuía anticorpos IgG contra o vírus. No município de Londrina, a frequência encontrada de $\operatorname{IgM}$ foi 1,2\% e de IgG $89 \%^{12}$, em São José do Rio Preto o IgM chegou a $0,6 \%$ e IgG, 93,1\% ${ }^{14}$; Campo Grande $0,1 \%$ para IgM e $87,9 \%$ para $\operatorname{IgG}^{13}$, no Estado de Sergipe $0,1 \%$ para $\operatorname{IgM}$ e 71,6\% para $\operatorname{IgG}^{15}$ e em Caxias do Sul a frequência de IgM encontrada foi de $1 \%{ }^{16}$, esses estudos utilizaram o método de ELISA para dosagem de anticorpos. No estudo realizado em Campo Grande a frequência de positividade para rubéola $\operatorname{IgG}$ mostrou relação com a faixa etária. $\mathrm{O}$ estudo aponta menor susceptibilidade à doença em pacientes com mais de 31 anos $^{13}$.

A alta taxa de gestantes com IgG positivo contra o vírus da rubéola no presente trabalho demonstra uma boa imunização nas mulheres do noroeste paranaense. Sugere-se que uma contínua e abrangente cobertura vacinal poderá resultar em baixa incidência dessa infecção em gestantes e, consequentemente, da síndrome da rubéola congênita (SRC) que ainda vem ocorrendo em algumas regiões do Brasil. Em 2008, 48 novos casos de SRC foram notificados no país e, em 2009, 15 casos $^{11}$. Isso pode ser alcançado a partir de campanhas de vacinação, efetivas estratégias para conscientização da população e monitoramento da rubéola através de um programa abrangente de triagem diagnóstica. Diante dos resultados obtidos, é possível sugerir que se mantenha a preconização do MS de sorologia para rubéola no pré-natal somente quando houver suspeita clínica.

Este estudo possui algumas limitações importantes, as quais incluem o curto período de coleta de dados, a ausência da informação do período gestacional da paciente e o delineamento do estudo, pois os estudos transversais não são os mais adequados para se investigar fatores de risco.

A frequência dessas doenças infecciosas em gestantes do noroeste do Paraná se mostrou compatível com outros estudos realizados no Brasil. Sugere-se que pesquisas sobre a prevalência e incidência destas doenças em gestantes sejam incentivadas, para possibilitar o planejamento de estratégias em prevenção, profilaxia e tratamento dessas infecções, evitando a sua transmissão vertical e os possíveis danos à saúde dos recém-nascidos, que, na maioria das vezes, provocam sequelas irreparáveis com consequente elevação de custos para o sistema de saúde.

\section{Referências}

1. Brasil. Ministério da Saúde. Secretaria de Atenção à Saúde. Departamento de Ações Programáticas Estratégicas. Área Técnica de Saúde da Mulher. Pré-natal e puerpério: atenção qualificada e humanizada: manual técnico. Brasília (DF): Ministério da Saúde; 2006.

2. UNAIDS World AIDS Day Report. Geneva: UNAIDS; 2011.

3. Xiao XM, Li AZ, Chen X, Zhu YK, Miao J. Prevention of vertical hepatitis $B$ transmission by hepatitis $B$ immunoglobulin in the third trimester of pregnancy. Int J Gynaecol Obstet. 2007;96(3): 167-70.

4. Piazza M, Urbanetz AA, Carvalho NS, Nascimento DJ. Hepatites virais e gestação. Diagn Tratamento. 2010;15(1):12-8.

5. Peyron F, Wallon M, Liou C, Garner P. Treatments for toxoplasmosis in pregnancy. Cochrane Database Syst Rev. 2000;(2):CD001684.

6. Brasil. Ministério da Saúde. Secretaria de Vigilância em Saúde. Programa Nacional de DST e Aids. Recomendações para profilaxia da transmissão vertical do HIV e terapia antirretroviral em gestantes. Brasília (DF): Ministério da Saúde; 2010.

7. Montoya JG, Remington JS. Management of Toxoplasma gondii infection during pregnancy. Clin Infect Dis. 2008;47(4):554-66.

8. Amendoeira MRR, Camillo-Coura LF. Uma breve revisão sobre toxoplasmose na gestação. Sci Med. 2010;20(1):113-9.

9. Margonato FB, Silva AMR, Soares DA, Amaral DA, Petris AJ. Toxoplasmose na gestação: diagnóstico, tratamento e importância de protocolo clínico. Rev Bras Saúde Matern Infant. $2007 ; 7(4): 381-6$.
10. Figueiró-Filho EA, Lopes AHA, Senefonte FRA, Souza Júnior VG, Botelho CA, Figueiredo MS, et al. Acute toxoplasmosis: study of the frequency, vertical tansmission rate and the relationship between maternal-fetal diagnostic tests during pregnancy in a Central-Western state of Brazil. Rev Bras Ginecol Obstet. 2005;27(8):442-9.

11. Brasil. Ministério da Saúde. Secretaria de Vigilância em Saúde. Departamento de Vigilância Epidemiológica. Guia de vigilância epidemiológica. 7a ed. Brasília (DF): Ministério da Saúde; 2009. Caderno 2: Rubéola; p. 1-17.

12. Reiche EMV, Morimoto HK, Farias GN, Hisatsugu KR, Geller L, Gomes ACLF, et al. Prevalência de tripanossomíase americana, sífilis, toxoplasmose, rubéola, hepatite $B$, hepatite $C$ e da infecção pelo vírus da imunodeficiência humana, avaliada por intermédio de testes sorológicos, em gestantes atendidas no período de 1996 a 1998 no Hospital Universitário Regional Norte do Paraná (Universidade Estadual de Londrina, Paraná, Brasil). Rev Soc Bras Med Trop. 2000;33(6):519-27.

13. Figueiró-Filho EA, Senefonte FRA, Lopes AHA, Morais OO, Souza Júnior VG, Maia TL, et al. Frequência das infecções pelo HIV-1, rubéola, sífilis, toxoplasmose, citomegalovírus, herpes simples, hepatite B, hepatite C, doença de Chagas e HTLV I/II em gestantes, do Estado de Mato Grosso do Sul. Rev Soc Bras Med Trop. 2007;40(2): 181-7.

14. Gonçalves MAS, Matos CCB, Spegiorin LCJF, Oliani DCMV, Oliani $\mathrm{AH}$, Mattos LC. Seropositivity rates for toxoplasmosis, rubella, syphilis, cytomegalovirus, hepatitis and HIV among pregnant women receiving care at a public health service, São Paulo State, Brazil. Braz J Infect Dis. 2010;14(6):601-5. 
15. Inagaki ADM, Oliveira LAR, Oliveira MFB, Santos RCS, Araújo RM, Alves JAB, et al. Soroprevalência de anticorpos para toxoplasmose, rubéola, citomegalovírus, sífilis e HIV em gestantes sergipanas. Rev Soc Bras Med Trop. 2009;42(5):532-36.

16. Madi JM, Souza RS, Araújo BF, Oliveira Filho PF, Rombaldi RL, Mitchell C, et al. Prevalence of toxoplasmosis, HIV, syphilis and rubella in a population of puerperal women using Whatman 903® filter paper. Braz J Infect Dis. 2010;14(1):24-9.

17. Machado Filho AC, Sardinha JFJ, Ponte RL, Costa EP, da Silva SS, Martinez-Espinosa FE. Prevalence of infection for HIV, HTLV, $\mathrm{HBV}$ and of syphilis and chlamydia in pregnant women in a tertiary health unit in the western Brazilian Amazon region. Rev Bras Ginecol Obstet. 2010;32(4):176-83.
18. Cardoso DDP, Faria EL, Azevedo MSP, Queiroz DAO, Martins RMB, Souza TT, et al. Soroepidemiologia para o vírus da hepatite B (VHB) em gestantes/parturientes e sua transmissão para recém-nascidos em Goiânia, GO. Rev Soc Bras Med Trop. 1996;29(4):349-53.

19. Arraes LC, Sampaio AS, Barreto S, Guilherme MAS, Lorenzato $F$. Prevalence of hepatitis $B$ in parturients and perinatal serologic profile. Rev Bras Ginecol Obstet. 2003;25(8):571-6.

20. Mioranza SL, Meireles LR, Mioranza EL, Andrade Júnior HF. Evidência sorológica da infecção aguda pelo Toxoplasma gondii em gestantes de Cascavel, Paraná. Rev Soc Bras Med Trop. 2008;41 (6):628-34.

21. Sartori AL, Minamisava R, Avelino MM, Martins CA. Triagem pré-natal para toxoplasmose e fatores associados à soropositividade de gestantes em Goiânia, Goiás. Rev Bras Ginecol Obstet. 201 1;33(2):93-8. 